\title{
CLIMA E COMPORTAMENTOS DE APRENDIZAGEM GRUPAL
}

\author{
Group learning climate and behaviors
}

\section{RESUMO}

Há um aumento do reconhecimento por parte das organizações da necessidade de reestruturar o trabalho em torno de equipes, proporcionando oportunidades para os membros aprenderem com os colegas por meio do diálogo e discussão. Este estudo teve como objetivo definir e operacionalizar o clima para a aprendizagem grupal (CAG) como um preditor dos comportamentos de aprendizagem grupal. CAG foi definido e operacionalizado como uma variável latente de segunda ordem com sete dimensões, de modo a obter validade de conteúdo, convergente e discriminante. A hipótese foi testada a partir de uma amostra de 217 trabalhadores que atuam em equipe, em uma organização de desenvolvimento e treinamento para o uso de softwares. A modelagem de equações estruturais revelou que o CAG tem forte relação com os comportamentos de aprendizagem grupal. Assim, a promoção de um CAG requer que a equipe perceba que tem: apoio da gestão; tempo suficiente para realizar o trabalho e aprender; controle sobre eventos organizacionais, possiblidade de iniciar uma ação e tomar decisões; oportunidades de aprender com os especialistas; acesso fácil a informações e orientações escritas e sentimento geral de satisfação com o local de trabalho.

Palavras-chave: Clima para a aprendizagem grupal. Comportamentos de Aprendizagem grupal. Modelo de equações estruturais. Viés do método.

\author{
Silvania da Silva Onça* \\ Diógenes de Souza Bido** \\ Adriana dos Santos Caparróz Carvalho ${ }^{\star * *}$
}

\section{ABSTRACT}

There is increased recognition by the organizations of the need to restructure work around teams, thus providing opportunities for members to learn from colleagues through dialogue and discussion. In this study, our purpose was to define and to operationalize the group learning climate (GLC) as a predictor of the group learning behavior. We defined and operationalized GLC as a second order latent variable with seven dimensions, in order to obtain content validity, both convergent and discriminant. We tested this hypothesis with a sample of 217 workers who work as a team in an organization that develops software and provides training courses in software use. The structural equation modeling showed that the GLC has a strong correlation with the group learning behavior. Thus, the promotion of the GLC requires that the team realize that they have: management support; enough time to do the work and learn; control over organizational events; the possibility to initiate action and make decisions; opportunities to learn from experts; easy access to information and written guidelines, and a general feeling of satisfaction with the workplace.

Keywords: Group learning climate. Group learning behavior. Structural equation modeling. Common method bias.

\footnotetext{
* Professora Adjunta da Universidade Federal do Sul e Sudeste do Pará - Instituto de Estudos em Saúde Biológica. Doutora em Administração de Empresas pela Universidade Presbiteriana Mackenzie. E-mail: silvaniaonca@ yahoo.com.br.

** Professor do Programa de Pós-Graduação em Administração de Empresas do Centro de Ciências Sociais e Aplicadas da Universidade Presbiteriana Mackenzie (PPGA/UPM). Doutor em Administração de Empresas pela Faculdade de Economia e Administração da Universidade de São Paulo (FEA/USP). E-mail: diogenesbido@ yahoo.com.br.

***Professora Adjunta da Universidade Federal do Mato Grosso - Faculdade de Administração e Ciências Contábeis. Doutora em Administração de Empresas pela Universidade Presbiteriana Mackenzie. E-mail: adrianacaparroz@ufmt.br.
} 


\section{INTRODUÇÃO}

A rápida evolução da tecnologia e dos sistemas de informação aumenta ainda mais a urgência de mudar e aprender no local de trabalho. Em um mundo assim, as competências individuais estabelecidas e as maneiras habituais de realizar o trabalho podem se tornar insuficientes para atender à constante inovação.

Por isso, há um aumento do reconhecimento por parte das organizações da necessidade de reestruturar o trabalho em torno de equipes que são definidas como indivíduos que atuam de modo interdependente para atingir objetivos comuns em um contexto organizacional mais amplo (KOZLOWSKI; ILGEN, 2006).

Conforme Janz (1999), ambientes orientados para o trabalho em equipe proporcionam oportunidades para os membros aprenderem com os colegas especialistas por meio do diálogo e discussão. 0 clima da equipe desempenha um papel central na determinação de comportamentos, atitudes e ações dos membros através da melhoria do ambiente psicológico positivo (BASAGLIA et al., 2010) e tem uma influência direta sobre a cognição da equipe (AÇIKGÖZ et al., 2013).

Comportamentos de aprendizagem no nível grupal dizem respeito aos processos de avaliar, agir e avaliar novamente, desenvolvendo um ciclo contínuo de ação e reflexão com foco interno à equipe (EDMONDSON, 1999).

O clima para a aprendizagem grupal é construído por líderes e outras pessoas-chave, que aprendem com a sua experiência, influenciam a aprendizagem de outras pessoas, e criam um ambiente de expectativas que molda e apoia os resultados desejados que, por sua vez, são medidos e recompensados (MARSICK; WATKINS, 2003). No presente estudo, o clima para a aprendizagem grupal foi definido, originalmente, como as percepções que as equipes têm sobre o ambiente de trabalho ser facilitador ou inibidor da aprendizagem.

Apesar do reconhecimento da importância da aprendizagem e do clima para a aprendizagem no local de trabalho, não se conhece a relação destes construtos no nível grupal, que é a lacuna que este estudo pretende preencher. A busca por artigos com os termos de busca "comportamentos de aprendizagem grupal" e "clima para a aprendizagem", em Português e Inglês, foi realizada nas bases Proquest, EBSCO, Portal de periódicos da Capes, SPEEL, Scielo e Google Scholar. A maioria dos artigos tratavam de pesquisas no Ensino, Educação, Pedagogia e ações em sala de aula, e nenhum deles, relacionou ambos os construtos no contexto de organizações.

Assim, este estudo teve como objetivo avaliar a influência do clima para a aprendizagem grupal sobre os comportamentos de aprendizagem grupal.

Dado o ineditismo, entende-se que a contribuição seja relevante para o campo de pesquisa da Aprendizagem nas organizações, e no contexto organizacional, os resultados desse estudo podem servir de alerta aos gestores para que promovam o clima para a aprendizagem grupal.

Este artigo contém mais cinco seções, a saber: as seções 2 e 3 apresentam as definições teóricas, antecedentes e consequentes dos construtos, a hipótese que orienta 0 estudo e o modelo estrutural. Na seção 4 são descritos os procedimentos metodológicos 
e na seção 5 são apresentados os resultados. A discussão e considerações finais estão na seção 6.

\section{COMPORTAMENTOS DE APRENDIZAGEM GRUPAL}

Aprendizagem Grupal (AG) é definida de diferentes formas. Do conjunto de definições localizadas, sete artigos denominam o construto como "aprendizagem em equipes" (KASL; MARSICK; DECHANT, 1997; EDMONDSON, 2002; TSJOSVOLD; TANG; WEST, 2004; KAYES; KAYES; KOLB, 2005; MCCARTTHY; GARAVAN, 2008; BRESÓ et al., 2008; KNAPP, 2010). Cinco artigos referem-se à AG como "comportamentos de aprendizagem em equipes" (EDMONDSON, 1999; GIBSON; VERMEULEN, 2003; RAES; BOON; KYNDT; DOCHY, 2015; SUN; TEH; LIN, 2016; VAN DER VEGT; BUNDERSON, 2005). E os demais autores, abordam o construto como "aprendizagem grupal" (ARGOTE; GRUENFELD; NAQUIN, 2001; BIDO et al., 2011); "processos de aprendizagem em equipes" (KOSTOPOULOS; SPANOS; PRASTACOS, 2011; VAN OFFENBEEK, 2001); "processos de aprendizagem coletiva" (EDMONDSON; BOHMER; PISANO, 2001, KNAPP, 2010); "estratégias de aprendizagem em equipes" (BRESMAN, 2005); "aprendizagem colaborativa" (VAN DEN BOSSCHE, 2010) e "aprendizagem contínua em equipes" (FRANCO; ALMEIDA, 2011; SESSA; LONDON, 2006).

Inseridos na perspectiva de AG como processos grupais, os comportamentos de AG têm sido estudados pelos autores citados a partir do pressuposto de que ocorrem por meio da ação e reflexão. Comportamentos de AG podem ser definidos como processos de avaliar, agir e avaliar novamente, descrevendo um ciclo contínuo de ação e reflexão com foco interno à equipe (EDMONDSON, 1999). Nesta dinâmica, os processos de aprendizagem, as condições que lhe dão suporte, os comportamentos de aprendizagem da equipe mudam conforme aprendem (ARGOTE; GRUENFELD; NAQUIN, 2001).

Identificados os comportamentos relativos à aprendizagem no nível da equipe com base na literatura, relatos de entrevistas e observação de seu ambiente de trabalho, Edmondson (1999) construiu um instrumento de mensuração da frequência da ocorrência de cinco comportamentos de AG: a) pesquisar; b) refletir; c) discutir os erros e os resultados inesperados de ações; d) buscar feedback e e) experimentar dentro e como uma equipe.

As assertivas deste instrumento relativas à ação da equipe são associadas a aspectos comportamentais, voltando-se à aquisição de informações pela equipe via experimentação, por meio de questionamentos, busca de feedback ou atividades que ampliam os limites. Já as assertivas relativas à reflexão, estão associadas a diálogos, conversações, negociações, construções, reconstrução e discussão entre os membros uma vez que estes comportamentos podem assim ser classificados devido à sua natureza cognitiva, compartiIhada entre os membros.

Tendo em vista a concepção de AG de Edmondson (1999), Savelsbergh et al. (2009) ampliaram o instrumento para oito comportamentos de AG: a) Coconstrução de sentido: são ações de conversação entre os membros da equipe, baseando-se, ou modificando o significado original oferecido em alguma forma para chegar a novos significados do trabalho colaborativo, que anteriormente não estavam disponíveis para a equipe; 
b) Exploração de diferentes perspectivas: ações de conversação dos membros da equipe com o objetivo de explorar, compartilhar conhecimentos, opiniões e perspectivas diferentes; c) Análise dos erros: a discussão e análise de erros para impedi-los; d) Comunicação de erros: compartilhamento dos erros coletivamente para impedir a reincidência; e) Reflexão sobre os processos: discussão coletiva dos objetivos da equipe, pressupostos, métodos de trabalho e estratégias, verificando se a equipe está fazendo as coisas certas e corretamente; f) Reflexão sobre os resultados: olhar para as experiências do passado ou futuras ações para avaliar e aprender com eles; g) Busca de feedback: a busca de feedback internamente entre os membros da equipe e externamente à equipe para refletir e, finalmente, h) Experimentação: fazer coletivamente as atividades e de forma diferente do que antes, avaliando as diferenças nos resultados.

Apesar de ter sido dado uma atenção especial na literatura à segurança psicológica da equipe como antecedente de comportamentos de AG, até o momento não se conhece a influência do clima para a aprendizagem grupal nestes comportamentos, sendo, este estudo, o pioneiro na investigação desta influência.

\section{CLIMA PARA A APRENDIZAGEM GRUPAL}

De acordo com Schein (1990), clima organizacional são as percepções, crenças e significados que resultam das percepções, interpretações e expectativas individuais sobre o ambiente organizacional e que afetam o comportamento.

Tido como uma manifestação direta da cultura organizacional (SCHEIN, 1985), clima e cultura organizacionais são conceitos relacionados (ILJINS; SKVARCIANY; GAILE-SARKANE, 2015) e de impacto na aprendizagem individual, grupal e organizacional (GUPTA; PYNGAVIL, 2012; MIKKELSEN; GRONHAUG, 1999). No entanto, há uma confusão entre os estudiosos sobre o que constitui clima e cultura organizacionais (GLISSON, 2000).

Segundo Glisson (2000), cultura organizacional tem sido definida por diferentes autores e a partir das perspectivas individuais, de sistemas sociais, comportamentos, percepções, estruturas sociais e processos. Van Maanen e Schein (1979), por exemplo, definem cultura organizacional como crenças compartilhadas, valores e normas de comportamento no local de trabalho. Já Schein (1985), compreende a cultura como um conjunto mais profundo de cognições e vínculos afetivos.

Segundo Jones e James (1979) e Ljins; Skvarciany; Gaile-Sarkane (2015), o clima organizacional emerge do contexto organizacional, postulando que o clima faz parte da cultura de uma organização. Visto da perspectiva de que seus membros podem atribuir significados diferentes para um conjunto de fatores ou eventos, é possível que um ambiente de trabalho possa ter um clima de segurança, de trabalho e, consequentemente, um clima que facilita ou dificulta a aprendizagem (MIKKELSEN; GRONHAUG, 1999).

No nível grupal, Edmondson (1999) argumentou que a percepção do clima interpessoal nas equipes em que trabalham é importante para compreender o trabalho em equipe e aprendizagem grupal nas organizações. A autora identificou que a segurança psicológica da equipe - clima que permite a contribuição voluntária de si mesmo, de ideias e ações, 
para o trabalho coletivo - é preditora de aprendizagem grupal. Isto pode ocorrer porque a segurança psicológica, segundo Schein (1985), ajuda as pessoas a superarem a atitude defensiva em relação à aprendizagem quando se sentem frustradas em suas expectativas, inibindo os comportamentos de aprendizagem grupal.

Mas, segurança psicológica, é um insumo para o clima interpessoal (EDMONDSON, 1999) e diferencia-se de clima para a aprendizagem grupal. Enquanto a segurança psicológica da equipe é a crença comum de que um grupo é seguro para assumir risco interpessoal (EDMONDSON, 1999; ORTEGA et al. , 2014), definimos o clima para a aprendizagem grupal como as percepções que as equipes têm sobre o ambiente de trabalho ser facilitador ou inibidor da aprendizagem.

Esta definição foi elaborada a partir da definição de clima para a aprendizagem organizacional de Mikkelsen e Gronhaug (1999) e Slater e Narver (1995), que se basearam em Bartram, Dixon e Kalogera (1996). Foram realizadas as devidas adaptações semânticas (do nível organizacional para o grupal) e a multidimensionalidade foi mantida, mas as dimensões também foram redefinidas com foco nas equipes.

a) Estilo e relações de gestão: as percepções da equipe sobre o apoio da gestão;

b) Tempo: as percepções da equipe sobre a necessidade de tempo para realizarem o trabalho e aprender;

c) Autonomia e responsabilidade: as percepções da equipe sobre o controle de eventos organizacionais, iniciando uma ação e tomando decisões;

d) Estilo da equipe: as percepções da equipe sobre oportunidades de aprender com colegas especialistas;

e) Oportunidade de desenvolvimento: as percepções da equipe sobre oportunidades para aprenderem novos postos de trabalho e fazerem uma variedade de tipos de trabalho no local de trabalho;

f) Orientações sobre como realizar o trabalho: as percepções da equipe sobre 0 acesso a informações e orientações relevantes escritas, e

g) Satisfação com o local de trabalho: as percepções de um sentimento de satisfação geral da equipe com o local de trabalho.

\subsection{CLIMA PARA A APRENDIZAGEM GRUPAL COMO ANTECEDENTE DOS COMPORTAMENTOS DE AG}

Como clima para a aprendizagem grupal é um construto que ainda não tinha sido operacionalizado, a adaptação a partir de Bartram, Dixon e Kalogera (1996), procurou garantir a validade de conteúdo seguindo as recomendações de Netemeyer, Bearden e Sharma (2003). Além da validade convergente e discriminante, que são tratadas nas seções dedicadas à metodologia e aos resultados, também é importante avaliar se a operacionalização do construto (clima para a aprendizagem grupal) consegue prever construtos que são teoricamente relacionados, ou seja, validade de critério (HINKIN, 1998). Assim, se a definição e operacionalização realizadas neste estudo estão corretas (validade e confia- 
bilidade), entende-se que a hipótese $\mathrm{H} 1$ deve ser sustentada pelos dados: quanto mais facilitador o clima para a aprendizagem grupal mais frequente serão os comportamentos de aprendizagem grupal.

A Figura 1 ilustra o modelo estrutural testado nesta pesquisa.

Figura 1 - Modelo estrutural

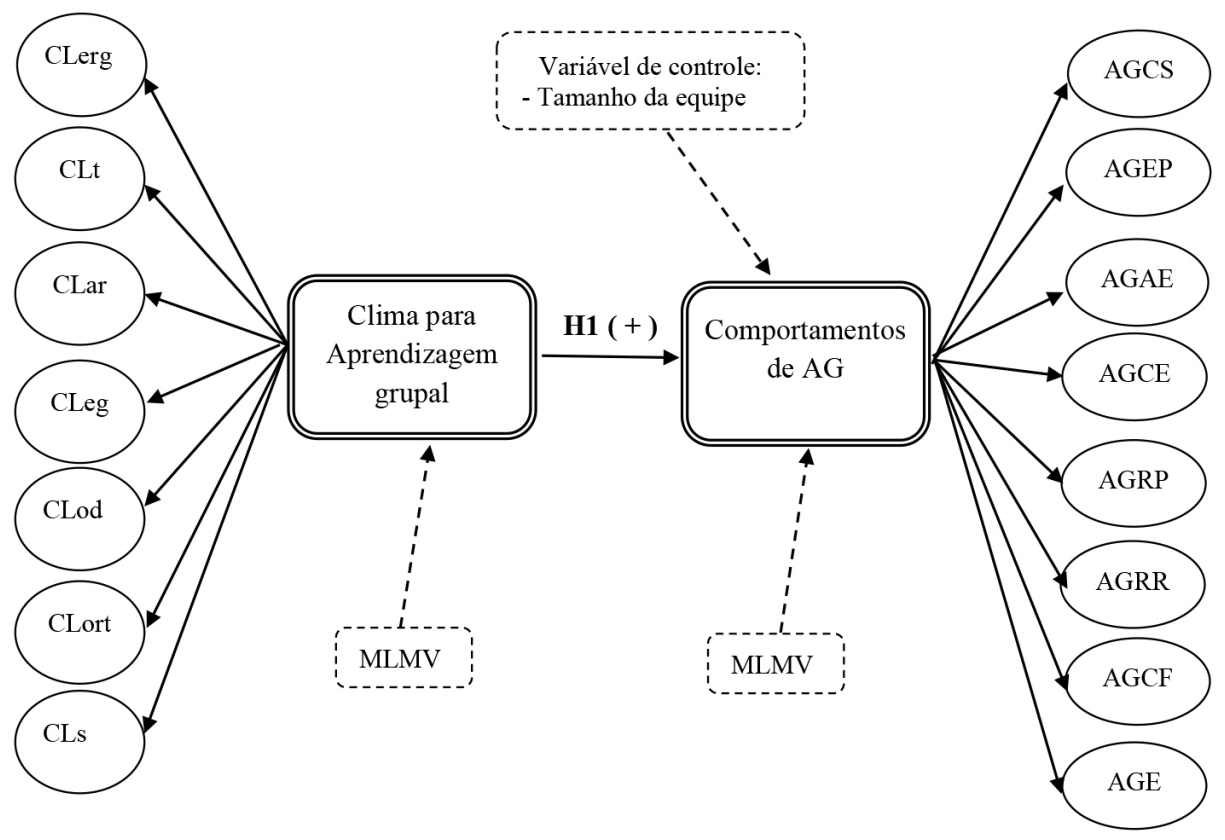

Fonte: Elaborado pelos autores.

Legenda: $C L e r g=$ Estilo e relação de gestão; $C L t=$ Tempo; CLar= Autonomia e responsabilidade; CLeg= Estilo de equipe; CLod= Oportunidade de desenvolvimento; CLort= Orientações sobre como realizar o trabalho; $\mathrm{CLS}=$ Satisfação com o local de trabalho; AGCS= Co-construção de sentido; AGEP= Exploração de diferentes perspectivas; $A G A E=$ Análise dos erros; $A G C E=$ Comunicação dos erros; $A G R P=$ Reflexões sobre processos; $A G R R=$ Reflexão sobre resultados; $A G C F=$ Comportamento de busca de feedback; $A G E=$ Experimentação. MLMV = measured latent marker variable, incluída no modelo para estimar e remover o viés do método (CHIN et al., 2013).

Nota: Para facilitar a visualização do modelo, foram omitidos os indicadores (resultados no Apêndice A). Comportamentos de AG e Clima para AG foram modeladas como variáveis latentes de segunda ordem.

Na Figura 1 a variável latente marcadora (MLMV - measured latent marker variable) foi incluída no modelo a fim de avaliar e eliminar o efeito do viés do método de acordo com as recomendações de Chin et al. (2013), o que está detalhado na seção 4.3, a seguir.

Quanto à variável de controle, tamanho da equipe, Puente-Palacios et al. (2011, p. 616) também realizaram estudo no nível de equipes e esta variável foi significante na previsão da satisfação no trabalho $(-0,18 ; p<0,01)$ e do comprometimento com a organização $(-0,14 ; p<0,01)$, apesar de na presente pesquisa a variável dependente ser outra, entendeu-se que o tamanho da equipe pode enviesar a relação que se pretende testar, por isso, decidiu-se por incluí-la no modelo, e para testar seu efeito o modelo foi analisado em três etapas, cujos resultados são apresentados na seção 5.3. 


\section{PROCEDIMENTOS METODOLÓGICOS}

As seções a seguir apresentam os procedimentos utilizados para a coleta e análise dos dados.

\subsection{INSTRUMENTO PARA A COLETA DE DADOS}

A mensuração dos comportamentos de aprendizagem grupal foi realizada por meio do Questionário de Aprendizagem Grupal de Savelsbergh et al. (2009), adaptado para a cultura brasileira por Onça e Bido (2015). A escala é composta por 28 itens relacionados a 8 dimensões: a) Co-construção de sentido - 3 itens; b) Exploração de diferentes perspectivas - 4 itens; c) Análise dos erros - 4 itens; d) Comunicação de erros - 4 itens; e) Reflexões sobre processos - 4 itens; f) Reflexão sobre resultados - 4 itens; g) Comportamento de busca de feedback - 2 itens; h) Experimentação - 3 itens, com respostas variando de 1 a 7 ("Nunca" a "Sempre").

Para a mensuração do clima para a aprendizagem grupal, além da adaptação para a cultura brasileira, foram realizadas adaptações semânticas nas assertivas do questionário de clima de aprendizagem organizacional de Bartram, Dixon e Kalogera (1996) com o objetivo de capturar o clima de aprendizagem no nível grupal. Originalmente, o questionário possuía dez itens para cada dimensão. São eles: a) Estilo e relação de gestão; b) Tempo; c) Autonomia e responsabilidade; d) Estilo de equipe; e) Oportunidade de desenvolvimento; f) Orientações sobre como realizar o trabalho e g) Satisfação com o local de trabalho. Com todos os itens reformulados e com respostas variando de 1 a 7 ("Discordo Totalmente" a "Concordo Totalmente") foram submetidos à análises estatísticas.

Para adaptação e validação para a cultura brasileira do Questionário de Clima para a aprendizagem foram realizadas traduções e consulta a especialistas no assunto (um doutor e três doutorandos) que realizaram modificações semânticas a fim de torná-lo compreensível e avaliaram a validade de conteúdo tendo em vista que as dimensões do construto foram redefinidas conceitualmente (de organizacional para grupal).

\subsection{COLETA DE DADOS}

Foram pesquisados os trabalhadores de uma empresa de desenvolvimento e treinamento para o uso de softwares que declararam trabalhar em equipe. Nas mensagens de apresentação e aplicação do formulário solicitou-se que as respostas fossem direcionadas à própria equipe de trabalho. Este procedimento é indicado porque a mensuração de variáveis no nível grupal requer que as questões sejam aplicadas aos membros, no entanto, deve-se estimular o respondente a responder conforme as percepções da equipe como um todo e não individualmente, em relação às variáveis (GUZZO et al., 1993).

Conseguiu-se uma amostra de 217 participantes de diferentes departamentos e níveis hierárquicos obtendo-se a taxa de retorno de 72,6\%. Pela análise de sensibilidade disponível no software G*Power 3 (FAUL et al., 2007): com esse tamanho de amostra, qualquer efeito superior a $3,5 \%$ seria detectado como significante a $5 \%$, com poder estatístico de $80 \%$. 


\subsection{ANÁLISE DE DADOS}

A análise dos dados permitiu verificar que não houve outliers, dados faltantes (missing values), nem erros de preenchimento do formulário. Desta forma, todas as respostas aos questionários foram mantidas para avaliação.

Primeiramente realizou-se a estatística descritiva das variáveis demográficas, seguida pela avaliação do modelo de mensuração por meio da análise fatorial confirmatória (validade convergente, validade discriminante e confiabilidade). 0 modelo estrutural foi estimado pelos mínimos quadrados parciais (PLS-PM - Partial Least Squares Path Modeling ou PLS-SEM - Partial Least Squares Structural Equation Modeling), dada a possibilidade de testar relações entre variáveis latentes sem a suposição de normalidade multivariada, capacidade de lidar com modelos complexos e o tamanho da amostra requerido ser muito menor do que aquele necessário para a estimação baseada em covariâncias, além de permitir o uso de indicadores formativos (HAIR Jr. et al., 2016), que é o caso do viés do método (MLMV - measured latent marker variable) no modelo testado.

\section{Viés do método (CMB - common method bias; CMV - common method variance)}

Conforme Podsakoff, Mackenzie e Podsakoff (2012) pode ocorrer viés do método (CMB) quando a variável dependente e as independentes são obtidas a partir da mesma fonte (respondente), resultando em uma subestimação ou superestimação da correlação entre essas variáveis latentes.

Quadro 1 - MLMV (Measured latent marker variable)

\begin{tabular}{|c|c|}
\hline Indicadores formativos & Referência \\
\hline $\begin{array}{c}\text { MLMV3. As pessoas ganham as recompensas } \\
\text { e punições que merecem. }\end{array}$ & $\begin{array}{l}\text { GOUVEIA, V.V. et al. Validade fatorial confirmatória e } \\
\text { consistência interna da Escala Global de Crenças no } \\
\text { Mundo Justo - GJWS, Interação em Psicologia, v.14, n.1, } \\
\text { p.21-29, 2010. }\end{array}$ \\
\hline $\begin{array}{l}\text { MLMV5. No que diz respeito à minha carreira, } \\
\text { quem toma as decisões sou eu. }\end{array}$ & $\begin{array}{l}\text { CORDEIRO, H.T.D.; CASADO, T.; LOPES, K.S.M. Validação } \\
\text { da escala de atitudes de carreira sem fronteiras e de } \\
\text { carreira proteana no Brasil. Anais ... XVI Semead, } 2013 .\end{array}$ \\
\hline $\begin{array}{l}\text { MLMV6. Sempre imagino como os produtos } \\
\text { serão no futuro }\end{array}$ & $\begin{array}{l}\text { GABRIEL, M.L.D.S.; SILVA, D.; MORETTI, S.L. O mito da } \\
\text { Geração Y: evidências a partir das relações de } \\
\text { causalidade entre idade e difusão e adoção de tecnologia } \\
\text { de estudantes universitários de São Paulo. Anais ... XVI } \\
\text { Semead } 2013 \text {. }\end{array}$ \\
\hline $\begin{array}{l}\text { MLMV7. Eu não compro produtos para minha } \\
\text { casa que prejudicam o meio ambiente. }\end{array}$ & $\begin{array}{l}\text { BRAGA JR., S.S. et al. Gestão socioambiental: uma } \\
\text { análise da consciência ecológica para o consumo "Verde" } \\
\text { no varejo supermercadista. Anais ... XIV Semead } 2011 .\end{array}$ \\
\hline
\end{tabular}

Fonte: É uma amostra dos nove itens usados como indicadores formativos da MLMV (CHIN, 2013). Respostas em escala de concordância (1 a 7 - DT/CT). 
Apesar das duas escalas usadas nesta pesquisa serem de sete pontos, a escala de Comportamentos de AG media frequência (1 a 7 nunca/sempre) e a escala de clima para AG media a concordância ( 1 a 7 concorda totalmente/discorda totalmente), o que é um fator positivo para amenizar o CMB.

Para avaliar e eliminar o CMB, seguiu-se as recomendações de Chin et al. (2013) incluindo uma variável latente (VL) com itens que não tinham nenhuma relação teórica com os demais construtos do modelo (domínio de conteúdo diverso). No Quadro 1 é apresentada uma amostra dos nove itens utilizados para mensurar este viés. Os resultados são apresentados na próxima seção.

\section{RESULTADOS}

A seguir são apresentados os resultados do estudo a partir das análises dos dados demográficos, avaliação do modelo de mensuração e avaliação do modelo estrutural.

\subsection{DADOS DEMOGRÁFICOS}

No momento da pesquisa a empresa contava com 299 colaboradores, dos quais, $217(72,6 \%)$ participaram e cujos dados demográficos são apresentados na Tabela 1.

Tabela 1 - Dados demográficos $(n=217)$

\begin{tabular}{lcc}
\hline Sexo & $\mathbf{n}$ & $\%$ \\
\hline Masculino & 123 & $57 \%$ \\
Feminino & 94 & $43 \%$ \\
\hline & & \\
\hline Idade & $\mathbf{n}$ & $\%$ \\
\hline 18 a 21 & 9 & $4 \%$ \\
22 a 25 & 54 & $25 \%$ \\
26 a 30 & 64 & $29 \%$ \\
31 a 35 & 47 & $22 \%$ \\
36 a 40 & 28 & $13 \%$ \\
41 a 50 & 15 & $7 \%$ \\
\hline & & \\
\hline Formação & $\mathbf{n}$ & $\%$ \\
\hline Ensino médio & 19 & $9 \%$ \\
Graduado & 122 & $56 \%$ \\
Especialista & 74 & $34 \%$ \\
Mestre & 1 & $0,5 \%$ \\
Doutor & 1 & $0,5 \%$ \\
\hline
\end{tabular}

\begin{tabular}{lcc}
\hline Tempo na empresa & $\mathbf{n}$ & $\mathbf{\%}$ \\
\hline menos de 6 meses & 25 & $12 \%$ \\
6 meses a 1 ano & 22 & $10 \%$ \\
acima de 1 ano até 4 & 96 & $44 \%$ \\
acima de 4 ano até 7 & 31 & $14 \%$ \\
acima de 7 ano até 10 & 16 & $7 \%$ \\
acima de 10 ano até 13 & 10 & $5 \%$ \\
acima de 13 ano até 16 & 10 & $5 \%$ \\
acima de 16 anos & 7 & $3 \%$ \\
\hline
\end{tabular}

\begin{tabular}{lcc}
\hline Tamanho da equipe & $\mathbf{n}$ & $\%$ \\
\hline até 2 pessoas & 19 & $9 \%$ \\
3 a 5 pessoas & 46 & $21 \%$ \\
6 a 10 pessoas & 61 & $28 \%$ \\
11 a 20 pessoas & 60 & $28 \%$ \\
mais de 20 pessoas & 31 & $14 \%$ \\
\hline
\end{tabular}

Fonte: Dados da pesquisa. 


\subsection{AVALIAÇÃO DO MODELO DE MENSURAÇÃO}

Todos os itens foram analisados como indicadores reflexivos (HAIR Jr. et al., 2016; MACKENZIE; PODSAKOFF; JARVIS, 2005) tendo em vista a definição dos construtos no referencial teórico. Foi realizada a AFC (Análise Fatorial Confirmatória) com todos os itens que mensuram os construtos conectados entre si conforme indica Brown (2006). O modelo foi estimado com o esquema de ponderação "factor" no software SmartPLS v.3.2.0 (RINGLE; WENDE; BECKER, 2015).

\section{Validade convergente}

Todos os itens de Comportamentos de Aprendizagem Grupal tiveram cargas significantes e acima de 0,6, como pode ser observado no Apêndice A. No entanto, dos 70 itens do Questionário de Clima para a Aprendizagem Grupal, 20 itens foram excluídos, obtendo-se assim, cargas acima 0,6. Isto pode ter ocorrido devido à adaptação cultural ou ao nível grupal, para as quais os itens sofreram alterações semânticas.

Elevada exclusão de itens do Questionário de Clima para a Aprendizagem no nível organizacional tem procedência na literatura: no estudo iraniano de Khanghahi e Jafari (2013) foram excluídos 28 itens do questionário por não apresentaram validade convergente ou discriminante adequados. A falta de validade discriminante de muitos itens do questionário também foi observada por Mikkelsen e Gronhaug (1999). Os dois estudos não apresentaram os itens que permaneceram e que foram excluídos, fato que dificulta uma comparação.

A validade convergente, no nível das variáveis latentes ( $V L)$, foi considerada adequada apesar dos indicadores de três VL apresentarem Variância Média Extraída (VME) abaixo de 0,5 (HAIR Jr. et al., 2016). São eles: CLar (Autonomia e responsabilidade) = 0,46; CLeg (Estilo de equipe) $=0,49$ e CLt (Tempo) =0,48. Na Tabela 2 é possível observar os valores de VME das VL avaliadas. Para aumentar a VME, bastaria excluir os itens com menores cargas fatorais, mas isto prejudicaria a validade de conteúdo e a replicabilidade futura da escala (DEVELLIS, 2003).

\section{Validade discriminante}

Todos os itens na matriz de cargas cruzadas (disponível com o primeiro autor) apresentaram cargas fatoriais mais altas em suas VL do que nas demais. Observa-se na Tabela 2 que a raiz quadrada da variância média extraída (valores na diagonal) foi maior do que as correlações (valores fora da diagonal) revelando haver validade discriminante, conforme Hair Jr. et al., (2016). 
Tabela 2 - Matriz de correlações entre as variáveis latentes de primeira ordem

\begin{tabular}{|c|c|c|c|c|c|c|c|c|}
\hline \multirow{2}{*}{$\begin{array}{l}\text { VL de } 1 \mathfrak{a} \\
\text { ordem }\end{array}$} & \multicolumn{8}{|c|}{ Comportamentos de Aprendizagem Grupal } \\
\hline & AGAE & AGCE & AGCF & AGCS & AGE & AGEP & AGRP & AGRR \\
\hline AGAE & 0,895 & & & & & & & \\
\hline AGCE & 0,862 & 0,907 & & & & & & \\
\hline AGCF & 0,539 & 0,563 & 0,868 & & & & & \\
\hline AGCS & 0,678 & 0,723 & 0,440 & 0,847 & & & & \\
\hline AGE & 0,626 & 0,623 & 0,604 & 0,535 & 0,907 & & & \\
\hline AGEP & 0,734 & 0,757 & 0,518 & 0,783 & 0,589 & 0,826 & & \\
\hline AGRP & 0,716 & 0,764 & 0,592 & 0,616 & 0,754 & 0,713 & 0,900 & \\
\hline AGRR & 0,785 & 0,780 & 0,659 & 0,673 & 0,770 & 0,729 & 0,839 & 0,913 \\
\hline CLar & 0,443 & 0,510 & 0,349 & 0,427 & 0,456 & 0,457 & 0,411 & 0,506 \\
\hline CLeg & 0,577 & 0,660 & 0,364 & 0,595 & 0,540 & 0,656 & 0,638 & 0,596 \\
\hline CLerg & 0,508 & 0,572 & 0,385 & 0,449 & 0,483 & 0,563 & 0,545 & 0,589 \\
\hline CLod & 0,503 & 0,553 & 0,441 & 0,403 & 0,579 & 0,532 & 0,514 & 0,613 \\
\hline CLort & 0,550 & 0,592 & 0,404 & 0,453 & 0,491 & 0,553 & 0,513 & 0,582 \\
\hline CLt & 0,451 & 0,504 & 0,383 & 0,399 & 0,431 & 0,470 & 0,472 & 0,542 \\
\hline CLs & 0,519 & 0,574 & 0,388 & 0,524 & 0,457 & 0,576 & 0,529 & 0,571 \\
\hline VME & 0,80 & 0,82 & 0,75 & 0,72 & 0,82 & 0,68 & 0,81 & 0,83 \\
\hline CC & 0,94 & 0,95 & 0,86 & 0,88 & 0,93 & 0,90 & 0,94 & 0,95 \\
\hline$A C$ & 0,92 & 0,93 & 0,67 & 0,80 & 0,89 & 0,84 & 0,92 & 0,93 \\
\hline
\end{tabular}

\begin{tabular}{|c|c|c|c|c|c|c|c|}
\hline \multirow{2}{*}{$\begin{array}{l}\text { VL de 1a } \\
\text { ordem }\end{array}$} & \multicolumn{7}{|c|}{ Clima para Aprendizagem Grupal } \\
\hline & CLar & CLeg & CLerg & CLod & CLort & CLt & CLs \\
\hline CLar & 0,681 & & & & & & \\
\hline CLeg & 0,448 & 0,700 & & & & & \\
\hline CLerg & 0,637 & 0,580 & 0,775 & & & & \\
\hline CLod & 0,630 & 0,556 & 0,681 & 0,735 & & & \\
\hline CLort & 0,605 & 0,604 & 0,679 & 0,664 & 0,716 & & \\
\hline CLt & 0,675 & 0,566 & 0,654 & 0,646 & 0,665 & 0,690 & \\
\hline CLs & 0,624 & 0,656 & 0,606 & 0,525 & 0,585 & 0,648 & 0,722 \\
\hline VME & 0,46 & 0,49 & 0,60 & 0,54 & 0,51 & 0,48 & 0,52 \\
\hline $\mathrm{CC}$ & 0,81 & 0,85 & 0,94 & 0,89 & 0,86 & 0,88 & 0,90 \\
\hline AC & 0,71 & 0,79 & 0,93 & 0,86 & 0,81 & 0,84 & 0,87 \\
\hline
\end{tabular}

Fonte: Elaborado pelos autores.

Legenda: $A G C S=$ Co-construção de sentido; $A G E P=$ Exploração de diferentes perspectivas; $A G A E=$ Análise dos erros; $A G C E=$ Comunicação dos erros; $A G R P=$ Reflexões sobre processos; $A G R R=$ Reflexão sobre resultados; $\mathrm{AGCF}=$ Comportamento de busca de feedback; $\mathrm{AGE}=$ Experimentação; CLerg= Estilo e relação de gestão; CLt= Tempo; CLar= Autonomia e responsabilidade; CLeg= Estilo de equipe; CLod=Oportunidade de desenvolvimento; CLort= Orientações sobre como realizar o trabalho; CLs= Satisfação com o local de trabalho; VME = Variância média extraída; CC = Confiabilidade composta; AC = Alfa de Cronbach.

Nota 1: Os valores na diagonal da matriz são a raiz quadrada da variância média extraída (VME), como estes valores são maiores do que os valores fora da diagonal (correlações) indica que há validade discriminante entre os construtos (HAIR Jr. et al., 2016).

Nota 2: Todos os construtos foram mensurados com escalas de 7 pontos ( 1 a 7 ).

Nota 3: Correlações iguais ou superiores a $|0,221|$ são significantes a $0,1 \%$. 


\section{Confiabilidade}

A confiabilidade também pode ser considerada adequada, com alfas de Cronbach entre 0,71 e 0,93 (Tabela 2), exceto a dimensão "Comportamento de busca de feedback" do construto AG que apresentou alfa de 0,67 , mas sua confiabilidade composta foi de 0,85 , que é a medida recomendada, quando se usa PLS-PM (HAIR JR. et al., 2016).

\section{Avaliação da mensuração das variáveis de segunda ordem}

Na Tabela 2 as células destacadas são correlações entre dimensões do mesmo construto. Em ambos os casos (Comportamentos de AG e Clima para a AG) era esperado que essas correlações fossem altas, porque foram modeladas como VL de $2^{a}$ ordem reflexivas. Para ambos os construtos a correlação média foi superior a 0,6, com valor mínimo de 0,44, o que é indicativo de validade convergente.

Tabela 3 - Matriz de correlações entre as variáveis latentes de primeira ordem

\begin{tabular}{ccc}
\hline VL de 2a ordem & Clima AG & Comportamentos AG \\
\hline Clima AG & $\mathbf{0 , 8 1 7}$ & \\
Comportamentos AG & 0,654 & $\mathbf{0 , 8 4 8}$ \\
\hline & & \\
\hline Variância média extraída & 0,67 & 0,72 \\
Confiabildiade composta & 0,93 & 0,95 \\
\hline
\end{tabular}

Fonte: Elaborado pelos autores.

Nota 1: Os valores na diagonal da matriz são a raiz quadrada da variância média extraída (VME), como estes valores são maiores do que os valores fora da diagonal (correlação) indica que há validade discriminante entre os construtos (HAIR Jr. et al., 2016).

Nota 2: Correlações iguais ou superiores a $|0,221|$ são significantes a $0,1 \%$.

Para modelar as VL de $2^{\mathrm{a}}$ ordem no SmartPLS os indicadores de suas VL de primeira ordem foram repetidos nas $\mathrm{VL}$ de $2^{\mathrm{a}}$ ordem, como é explicado por Wetzels et al. (2009) e Hair Jr. et al. (2016), assim, as relações entre as VL de $2^{a}$ ordem e suas VL de $1^{\text {a }}$ ordem são interpretadas como cargas fatoriais, que são usadas para se calcular a VME e a confiabilidade composta. Na Tabela 3, observa-se que há validade convergente, discriminante e confiabilidade neste nível também ( $2^{\mathrm{a}}$ ordem).

\subsection{AVALIAÇ̃̃O DO MODELO ESTRUTURAL}

A estimação do modelo estrutural foi complexa por envolver variáveis de segunda ordem (Comportamentos de AG e Clima para a AG), inclusão de variável de controle (tama- 
nho da equipe) e avaliação e controle do viés do método. Por isso, o modelo foi analisado em três etapas (Tabela 4), cujos resultados serão comentados nas próximas seções.

\section{Teste da hipótese}

A Tabela 4 apresenta os resultados de três modelos estruturais, que foram necessários para se avaliar os efeitos da variável de controle (tamanho da equipe) e do viés do método (CMB - common method bias).

Em nenhum dos modelos houve problemas de multicolinearidade, já que o valor do VIF (fator de inflação da variância) está abaixo de 5 (HAIR JR. et al., 2016), o tamanho da equipe não teve relação significante com os comportamentos de $A G$, e o viés do método foi estimado em 0,078, ou seja, apesar de haver uma superestimação do coeficiente estrutural, esse viés é pequeno, e o modelo 3 apresenta o coeficiente estrutural $(0,654 ; p<0,001)$ não viesado, que suporta a hipótese H1. Para avaliar quanto da variância dos comportamentos de AG é explicada apenas pelo clima para AG, o beta do modelo 3 foi multiplicado pela correlação (TENENHAUS et al., 2005), resultando em 47\%.

Tabela 4 - Modelos estruturais preditores dos Comportamentos de AG

\begin{tabular}{lcc|ccc|ccc|c}
\hline \multirow{2}{*}{ Preditores } & \multicolumn{2}{c|}{ Modelo 1 } & \multicolumn{3}{|c|}{ Modelo 2 } & \multicolumn{3}{c|}{ Modelo 3 } & Diferença \\
& $\boldsymbol{\beta}$ & $\mathbf{p}$ & $\boldsymbol{\beta}$ & $\mathbf{p}$ & VIF & $\boldsymbol{\beta}$ & $\mathbf{p}$ & VIF & $\boldsymbol{\beta}$ 2 - $\mathbf{\beta 3}$ \\
\hline Tamanho da equipe & $-0,011$ & 0,874 & 0,064 & 0,152 & 1,011 & 0,075 & 0,089 & 1,018 & \\
MLMV & & & & & 0,142 & 0,018 & 1,467 & \\
H1(+) Clima AG & & 0,732 & 0,000 & 1,011 & 0,654 & 0,000 & 1,458 & 0,078 \\
\hline $\mathbf{R}^{\mathbf{2}}$ & $0,0 \%$ & $53,0 \%$ & & $54,5 \%$ & & \\
$\mathbf{R}^{\mathbf{2}}$ ajustado & $0,0 \%$ & $52,6 \%$ & & $53,8 \%$ & & \\
\hline
\end{tabular}

Fonte: Elaborado pelos autores.

Legenda: MLMV - measured latent marker variable é a variável que foi incluída no modelo para estimar e remover o efeito do CMB. No modelo 3 ela acrescentada como é explicado em Chin (2013), assim, os resultados apresentados são coeficientes estruturais padronizados após a remoção do efeito do CMB.

Nota 1: Todos os modelos estruturais foram estimados no software SmartPLS 3.2.0, e os valores-p foram estimados por bootstrap com 500 reamostragens e a opção no sign changes.

\section{DISCUSSÃO DOS RESULTADOS E CONSIDERAÇÕES FINAIS}

O objetivo deste estudo foi avaliar a influência do clima para a aprendizagem grupal sobre os comportamentos de aprendizagem grupal. A hipótese foi suportada pelos dados, de modo que o clima para a AG explica $47 \%$ dos comportamentos de AG. Assim, elevados níveis do clima para a aprendizagem em equipes de trabalho elevam a possibilidade de seus membros agirem e refletirem coletivamente, o que são características de comportamentos de aprendizagem grupal. 
O resultado está em conformidade com a afirmativa de Mapuring e Magni (2012) de que o clima que apoia e valoriza a experimentação e o diálogo pode ser propício para a exploração das tecnologias de informação (lembrando que os entrevistados são de empresa de desenvolvimento e treinamento para o uso de softwares) e, consequentemente, à aprendizagem grupal. Isto pode ocorrer porque as atitudes dos empregados em direção a um novo sistema podem ser moldadas pelas atitudes de seus colegas de trabalho influenciando a intenção de usar a tecnologia (GALLETTA et al., 1995).

Um clima favorável à aprendizagem grupal requer um trabalho de coordenação e cooperação entre os membros da equipe, conforme assinalou Wageman (1995), para que influencie o comportamento dos funcionários referente ao uso da tecnologia (GALLIVAN; SPITLER; KOUFARIS, 2005; LIANG et al., 2003) e compartilhamento de informações (GLOMB; LIAO, 2003).

Alavancar um clima de aprendizagem grupal requer que a equipe perceba apoio da gestão; tempo suficiente para realizar o trabalho e aprender; controle sobre eventos organizacionais, que pode iniciar uma ação e tomar decisões; oportunidades de aprender com os especialistas; oportunidades de aprender novos postos de trabalho e que pode fazer uma variedade de trabalhos no local de trabalho; acesso fácil a informações e orientações escritas e sentimento geral de satisfação com o local de trabalho. De acordo com os resultados deste estudo, tais percepções são importantes para a promoção de comportamentos de aprendizagem grupal. Em contraste, com baixas percepções destes itens por parte da equipe, pode ser baixa a intenção da equipe de desenvolver comportamentos de aprendizagem grupal.

Elevados níveis de clima para a aprendizagem grupal podem ser estimulados por meio de práticas de gestão promotoras de trabalho em equipe. A coordenação e cooperação entre os membros da equipe são indispensáveis para o diálogo que busca ou modifica o sentido do trabalho; para a exploração, compartilhamento de conhecimentos, opiniões e diferentes perspectivas; comunicação e análise dos erros para impedi-los; reflexão sobre os processos e resultados; busca de feedback e experimentação.

Alavancar o clima de aprendizagem grupal é especialmente importante para a aprendizagem grupal devido às exigências para a adoção de novas tecnologias e sistemas de informação. Em organizações de desenvolvimento e treinamento para o uso softwares um elevado clima para a aprendizagem grupal é especialmente importante quando adotam o trabalho em equipe.

O tamanho da equipe não se relacionou com os comportamentos de aprendizagem grupal, de certo modo, este resultado era esperado porque na pesquisa de Ortega et al. (2014) a correlação foi de apenas $-0,20(p<0,05)$, mas a inclusão do tamanho da equipe no modelo teve o principal objetivo de eliminar explicações alternativas, ou seja, realizar o controle estatístico.

Considera-se, do ponto de vista metodológico, que o estudo contribui com: (i) desenvolvimento e validação da escala de clima para a AG (com sete fatores) que estão disponíveis no apêndice $A$; (ii) implementação do procedimento recomendado por Chin et al. (2013) com o objetivo de eliminar o viés do método comum de coletas de dados.

Como limitação do estudo, tem-se: (i) a impossibilidade de generalização dos resultados uma vez que a amostra não é probabilística; (ii) a imprecisão da mensuração, pois 
o indivíduo respondeu pela equipe, para futuras pesquisas, se houver a possibilidade de obter dados de várias pessoas de cada equipe (agregando as respostas individuais no nível grupal), minimizaria este problema.

Outras sugestões para estudos futuros são: (i) desenvolver um estudo longitudinal para identificar mudanças nas percepções relativas ao clima de AG e nos comportamentos de AG; (ii) para aumentar a precisão da mensuração e reduzir vieses de memória, os itens do questionário poderiam ser mais específicos (Aprender o quê? Quando?), convidando o respondente a indicar sua percepção sobre uma competência específica, em um intervalo temporal definido, (iii) nesta pesquisa assumiu-se grupos e equipes como sinônimos e formalizados na organização, porém, Weber e Grisci (2013) apresentam uma análise crítica desses conceitos mostrando diferenças do discurso (equipes formalizadas) para as redes informais, que, de fato apoiam tecnicamente e afetivamente seus membros, assim, a tarefa de promoção do clima para a aprendizagem grupal pode ser entendido em maior profundidade a partir de pesquisas qualitativas que procurem mapear de que forma as dimensões do clima para a aprendizagem grupal interagem com as equipes formais e com as redes informais dentro da organização.

\section{REFERÊNCIAS}

AÇIKGÖZ, A. et al. Team Climate, Team Cognition, Team Intuition, and Software Quality: The Moderating Role of Project Complexity. Group Decision and Negotiation, v.23, n.4, p.1145-1176, 2013.

ARGOTE, L.; GRUENFELD, D. H.; NAQUIN, C. Group learning in organizations. In: TURNER, M. (ed.). Groups at Work: Advances in Theory and Researchs. Mahway, NJ: Erlbaum, 2001, p.369-411.

BARTRAM, D.; DIXON, A.; KALOGERA, S. The learning climate questionnaire: Shared perceptions within work groups. In: OCCUPATIONAL PSYCHOLOGY CONFERENCE, 1996. Proceedings... Eastbourne: The British Psychological Society, 1996, p.241-246.

BASAGLIA, S. et al. It knowledge integration capability and team performance: the role of team climate. The International Journal of Information Management, v. 30, n.1, p.542551, 2010.

BIDO, D. S. et al. Examinando a relação entre aprendizagem individual, grupal e organizacional em uma instituição financeira. REAd - Revista Eletrônica de Administração, v. 17, n. 1, p. 58-86, 2011.

BRESMAN, $\mathrm{H}$. Learning strategies and performance in organizational teams. Massachusetts Institute of Technology, 2005.

BRESÓ, I. et al. Development and validation of the Team Learning Questionnaire. Comportamento Organizacional e Gestão, v.14, n.2, p.145-160, 2008.

BROWN, T. A. Confirmatory Factor Analysis for Applied Research. New York: The Guilford Press, 2006. 
CHIN, W. W. et al. Controlling for common method variance in PLS analysis: the measured latent marker variable approach. In: ABDI, H.; CHIN, W. W. et al. (ed.). New Perspectives in Partial Least Squares and Related Methods. New York: Springer, 2013. p.231-239.

DEVELLIS, R. F. Scale Development: theory and applications. 2nd ed. Thousand Oaks: Sage Publications, Inc., 2003.

EDMONDSON, A. C. Psychological safety and learning behavior in work teams. Administrative Science Quarterly, v.44, n.2, p.350-383, 1999.

. The local and variegated nature of learning in organizations. Organization Science, v.13, n.2, p.128-146, 2002.

EDMONDSON, A. C.; BOHMER, R. M.; PISANO, G. P. Disrupted routines: Team learning and new technology implementation in hospitals. Administrative Science Quarterly, v.46, n4, p.685-716, 2001.

FAUL, F. et al. G*Power 3: a flexible statistical power analysis program for the social, behavioral, and biomedical sciences. Behavior Research Methods, v. 39, n. 2, p. 175-91, 2007.

FRANCO, M.; ALMEIDA, J. Organisational learning and leadership styles in healthcare organisations: An exploratory case study. Leadership \& Organization Development Journal, v. 32, n. 8, p. 782-806, 2011.

GALLETTA, D.F. et al. Social influence and user training. Communications of the ACM, v. 38, n. 7, p.70-79, 1995.

GALLIVAN, M. J.; SPITLER, V. K.; KOUFARIS, M. Does information technology training really matter? A social information processing analysis of coworkers' influence on IT usage in the workplace. Journal of Management Information Systems, v. 22, n. 1, p. 153-192, 2005.

GUZZO, R. A. et al. Potency in groups: articulating a construct. British Journal of Psychology, v. 32, n. 1, p. 82-106, 1993.

GIBSON, C. B.; VERMEULEN, F. A healthy divide: subgroups as a stimulus for team learning behavior. Administrative Science Quarterly, v. 48, n. 2, p. 202-239, 2003.

GLISSON, C. Organizational climate and culture. In: PATTI, R. J. (ed.), The Handbook of Social Welfare Management. Thousand Oaks: Sage Publications, Inc, 2000, p. 195-218.

GLOMB, T. M.; LIAO, H. Interpersonal aggression in work groups: Social influence, reciprocal, and individual effects. Academy of Management Journal, v. 46, n. 4, p.486-496, 2003.

GUPTA, M. L.; PYNGAVIL, R. S. Effective Team Building in Relation to Organizational Culture and Organizational Climate in Banking Sector: An Inter-Correlation Analysis. Prestige International Journal of Management \& IT-Sanchayan, v. 1, n. 1, p. 52-66, 2012.

HAIR JR., J. F. et al. Primer on Partial Least Squares Structural Equation Modeling (PLS-SEM). $2^{a}$ ed. Thousand Oaks, CA: Sage Publications, Inc., 2016.

HINKIN, T. R. A Brief Tutorial on the Development of Measures for Use in Survey Questionnaires. Organizational Research Methods, v. 1, n. 1, p. 104-121, 1998. 
ILJINS, J.; SKVARCIANY, V.; GAILE-SARKANE, E. Impact on Organizational Climate trough Organizational Culture factors. Case Study of Latvia and Lithuania. Trends Economics and Management, v. IX, n. 24, p. 9-17, 2015.

JANZ, B. D. Self-directed teams in IS: correlates for improved systems development work outcomes. Information and Management, v. 35, n. 3, v. 171-192, 1999.

JONES, A. P.; JAMES, L. R. Psychological climate: dimensions and relationships of individuals and aggregated work environment perceptions. Organizational Behavior and Human Performance, v. 23, p. 201-250, 1979.

KASL, E.; MARSICK, V. J.; DECHANT, K. Teams as learners: a research-based model of team learning. The Journal of Applied Behavioral Science, v. 33, n. 2, p. 227-246, 1997.

KAYES, A. B.; KAYES, D. C.; KOLB, D. A. Experiential learning in teams. Simulation and Gaming. v. 6, n. 3, p. 330-354, 2005.

KHANGHAHI, M. E.; JAFARI, P. Model for organizational intelligence in Islamic Azad university (Zone 8). Mathematics Education Trends and Research, v. 2013, p.1-10, 2013.

KNAPP, R. Collective (team) learning process models: a conceptual review. Human Resource Development Review, v. 9, n. 1, p. 285-30, 2010.

KOSTOPOULOS, K. C.; SPANOS, Y. E.; PRASTACOS, G. P. Structure and Function of Team Learning Emergence: A Multilevel Empirical Validation. Journal of Management, v. 39, n. 6, p. 1430-1461, 2011.

KOZLOWSKI, S. W. J.; ILGEN, D. R. I. Enhancing the effectiveness of work groups and teams. Psychological Science in the Public Interest, v. 7, p. 77-124, 2006.

KOZLOWSKI, S. W. J.; BELL, B. S. Work groups and teams in organizations. In: BORMAN, W. C.; ILGEN, D. R.; KLIMOSKI, R. J. (Eds.). Handbook of psychology: Industrial and organizational psychology, 2003, p.333-375.

LIANG, H. et al. Understanding the influence of team climate on IT use. Journal of the Association for Information Systems, v. 11, n. 8, p. 414-432, 2010.

MCCARTHY, A.; GARAVAN, T. N. Team learning and metacognition: A neglected area of HRD Research and practice. Advances in Developing Human Resources, v. 10, n. 4, p. 509-524, 2008.

MACKENZIE, S. B.; PODSAKOFF, P. M.; JARVIS, C. B. The problem of measurement model misspecification in behavioral and organizational research and some recommended solutions. The Journal of Applied Psychology, v. 90, n. 4, p. 710-30, 2005.

MAPURING, L. M.; MAGNI, M. What's the weather like? The effect of team learning climate, empowerment climate, and gender on individuals' technology exploration and use. Journal of Management Information Systems, v. 29, n. 1, p. 79-113, 2012.

MARSICK, V.; WATKINS, K. E. Demonstrating the value of an organization's learning culture: the dimensions of the learning organization questionnaire. Advances in Developing Human Resources, v. 5, n. 2, p. 132-151, 2003. 
MIKKELSEN; A.; GRONHAUG, K. Measuring organizational learning climate: A cross-national replication and instrument validation study among public sector employees. Review of Public Personnel Administration, v. 19, n. 1, p. 31-44, 1999.

NETEMEYER, R. G.; BEARDEN, W. O.; SHARMA, S. Scaling procedures: issues and applications. Thousand Oaks: Sage Publications, 2003.

ONÇA, S. S.; BIDO, D. S. Bases de Poder do Gestor e Conflitos Intragrupais como antecedentes da Aprendizagem Grupal. In: ENCONTRO DA ANPAD, 39, Belo Horizonte, 2015. Anais... Belo Horizonte, 2015.

ORTEGA, A. et al. The Influence of Change-Oriented Leadership and Psychological Safety on Team Learning in Healthcare Teams. Journal of Business and Psychology, v. 29, n. 2, p. 311-321, 2014.

PODSAKOFF, P. M.; MACKENZIE, S. B.; PODSAKOFF, N. P. Sources of method bias in social science research and recommendations on how to control it. Annual Review of Psychology, v. 63, p. 539-569, 2012.

PUENTE-PALACIOS, K.; ALMEIDA, R. S.; REZENDE, D. V. O impacto da interdependência no trabalho sobre a efetividade de equipes. O\&S - Organizações \& Sociedade, v.18, n.59, p. 605-623, 2011.

RAES, E. et al. Measuring team learning behaviours through observing verbal team interaction. Journal of Workplace Learning, v. 27, n. 7, p. 476-500, 2015.

RINGLE, C. M.; WENDE, S.; BECKER, J. M. SmartPLS 3. Boenningstedt: SmartPLS GmbH, $<$ http://www.smartpls.com>. 2015.

SAVELSBERGH, C.; VAN DER HEIJDEN, B. I. J. M.; POELL, R. F. The development and empirical validation of a multidimensional measurement instrument for team learning behaviors. Small Group Research, v.40, n.5, p.578-607, 2009.

SCHEIN, E. H. Organizational culture and leadership: a dynamic view. San Francisco, Jossey Bass, 1985.

. Organizational culture. American Psychologist, v. 45, n. 2, p. 109-119, 1990.

SESSA, V. I.; LONDON, M. Continuous learning in organizations. Individual, group and organizational perspectives. New Jersey: Lawrence Erlbaum Associates, 2006.

SLATER, S. F.; NARVER, J. C. Market orientation and the learning organization. Journal of Marketing, v.59, n.1, p.63-74, 1995.

SUN, H. et al. Team Diversity, Learning, and Innovation: A Mediation Model. Journal of Computer Information Systems, v. 4417, n. July, p. 1-9, 2016.

TSJOSVOLD, D.; TANG, M. M. L.; WEST, M. Reflexity for team innovation in China: The contribution of goal interdependence. Group and Organization Management, v. 29, n. 5, p. 540-559, 2004.

VAN OFFENBEEK, M. Processes and Outcomes of Team Learning. European Journal of Work and Organizational Psychology, v. 10, n. 3, p. 303-317, 2001. 
VAN DEN BOSSCHE, P. et al. Team learning: building shared mental models. Instructional Science, v.39, n.3, p.283-301, 2010.

VAN DER VEGT, G. S.; BUNDERSON, J. S. Learning and performance in multidisciplinary teams: the importance of collective team identification. Academy of Management Journal, v. 48 , n. 3 , p. 532-547, 2005.

VAN MAANEN, J.; SCHEIN, E. H. Toward a theory of organizational socialization. In: STAW, B. M.; CUMMINGS, L. L. (eds.). Research in Organizational Behavior. JAI Press: Greenwich, 1979, p. 209-264.

TENENHAUS, M. et al. PLS path modeling. Computational Statistics \& Data Analysis, v. 48, n. 1, p. 159-205, 2005.

WAGEMAN, R. Interdependence and group effectiveness. Administrative Science Quarterly, v. 40, n. 1, p. 145-180, 1995.

WEBER, L.; GRISCI, C. L. I. Conexões entre trabalhadores: alternativas para além do discurso hegemônico de grupos e equipes. O\&S - Organizações \& Sociedade, v. 20, n. 65, p. 207-224, 2013.

WETZELS, M.; ODEKERKEN-SCHRÖDER, G.; OPPEN, C. VAN. Using PLS path modeling for assessing hierarchical construct models: guidelines and empirical illustration. MIS Quarterly, v. 33, n. 1, p. 177-195, 2009.

Data de submissão: 21/06/2016.

Data de aprovação: 06/03/2017. 


\section{APÊNDICE A}

\section{ITENS E CARGAS FATORIAIS}

Não foram apresentados todos os itens por limitação de espaço, mas estão disponíveis com o primeiro autor.

Comportamentos de aprendizagem grupal: Os itens tratam sobre o que A SUA EQUIPE DE TRABALHO FAZ para adquirir novos conhecimentos e habilidades, e NÃO sobre o que você faz, isoladamente. Para responder cada questão, utilize a escala: de 1 a 7 ("Nunca" a "Sempre").

\begin{tabular}{|c|c|}
\hline Variáveis latentes e uma amostra de seus indicadores & Carga Fatorial \\
\hline \multicolumn{2}{|l|}{ Coconstrução de sentido ( 3 itens, cargas de 0,699 a 0,915 ) } \\
\hline $\begin{array}{l}\text { AGCS1. Informações de membros da equipe são complementadas com informações } \\
\text { de outros membros do grupo. }\end{array}$ & 0,699 \\
\hline $\begin{array}{l}\text { AGCS2. Membros da equipe chegam a conclusões coletivamente, a partir das ideias } \\
\text { que são discutidas no grupo. }\end{array}$ & 0,909 \\
\hline \multicolumn{2}{|l|}{ Exploração de diferentes perspectivas ( 4 itens, cargas de 0,780 a 0,869 ) } \\
\hline AGEP4. Membros da equipe escutam cuidadosamente uns aos outros. & 0,814 \\
\hline AGEP5. Se algo não está claro, nós questionamos uns aos outros. & 0,78 \\
\hline \multicolumn{2}{|l|}{ Análise dos erros ( 4 itens, cargas de 0,865 a 0,917 ) } \\
\hline AGAE8. Depois de um erro, a equipe analisa em conjunto qual foi a causa. & 0,896 \\
\hline AGAE9. Na minha equipe de trabalho, nós acreditamos que é útil analisar os erros. & 0,865 \\
\hline \multicolumn{2}{|l|}{ Comunicação de erros ( 4 itens, cargas de 0,861 a 0,946 ) } \\
\hline $\begin{array}{l}\text { AGCE12. Os membros da equipe grupo comunicam seus erros, para evitar que outros } \\
\text { cometam o mesmo erro. }\end{array}$ & 0,861 \\
\hline $\begin{array}{l}\text { AGCE13. Nós discutimos sobre os erros com nossa equipe, porque erros e suas } \\
\text { soluções podem transmitir informações importantes. }\end{array}$ & 0,946 \\
\hline \multicolumn{2}{|l|}{ Reflexões sobre processos ( 4 itens, cargas de 0,881 a 0,925 ) } \\
\hline AGRP16. Nós frequentemente discutimos sobre os métodos de trabalho da nossa & 0,883 \\
\hline $\begin{array}{l}\text { AGRP17. Como uma equipe, nós discutimos regularmente sobre a eficácia da nossa } \\
\text { colaboração. }\end{array}$ & 0,911 \\
\hline \multicolumn{2}{|l|}{ Reflexão sobre resultados ( 4 itens, cargas de 0,844 a 0,946 ) } \\
\hline $\begin{array}{l}\text { AGRR20. Em nossa equipe, nós examinamos o que podemos aprender a partir de } \\
\text { nossas conquistas. }\end{array}$ & 0,913 \\
\hline $\begin{array}{l}\text { AGRR21. Em nossa equipe, nós verificamos se nossas ações provocaram o que nós } \\
\text { esperávamos inicialmente. }\end{array}$ & 0,944 \\
\hline \multicolumn{2}{|l|}{ Comportamento de busca de feedback ( 2 itens) } \\
\hline AGCF24. Nós analisamos nosso desempenho de acordo com outras equipes. & 0,885 \\
\hline $\begin{array}{l}\text { AGCF25. Nós pedimos feedback interno e externo das partes interessadas nos nossos } \\
\text { resultados. }\end{array}$ & 0,851 \\
\hline \multicolumn{2}{|l|}{ Experimentação ( 3 itens, cargas de 0,855 a 0,941 ) } \\
\hline AGE26. Em nossa equipe, nós temos experiência com outros métodos de trabalho. & 0,855 \\
\hline AGE27. Nossa equipe testa novos métodos de trabalho. & 0,941 \\
\hline
\end{tabular}


A seguir você encontrará uma série de sentenças que procuram descrever o clima para aprendizagem para sua EQUIPE DE TRABALHO. Por favor, utilize a escala abaixo para apontar a frequência com que as situações descritas ocorrem em SUA EQUIPE DE TRABALHO, registrando a sua resposta na coluna específica ao lado. Use os seguintes números para as suas respostas: 1 a 7 ("Discordo Totalmente" a "Concordo Totalmente").

\begin{tabular}{lc}
\hline Variáveis latentes e uma amostra de seus indicadores & Carga Fatorial \\
\hline $\begin{array}{l}\text { Estilo e relação de gestão (10 itens: cargas de } 0,702 \text { a 0,826) } \\
\text { CLerg1. O superior imediato da minha equipe nos faz sentir um membro valioso da } \\
\text { equipe. }\end{array}$ & 0,818 \\
$\begin{array}{l}\text { CLerg8. Nós (membros da equipe) podemos discutir sobre trabalho com nosso } \\
\text { superior imediato e receber comentários construtivos. }\end{array}$ & 0,766 \\
\hline & \\
\hline $\begin{array}{l}\text { Tempo (10 itens: } 2 \text { excluídos e demais com cargas de 0,609 a 0,748) } \\
\text { CLt9. Não há tempo para praticar as coisas que nós (membros da equipe) precisamos }\end{array}$ & 0,659 \\
$\begin{array}{l}\text { saber fazer. } \\
\text { CLt30. Se nós (membros da equipe) temos uma tarefa difícil, nós temos tempo para } \\
\text { sentar e pensar sobre como nós iremos executá-la. }\end{array}$ & 0,712 \\
\hline
\end{tabular}

Autonomia e responsabilidade (10 itens: 5 excluídos e demais com cargas de 0,635 a

0,785 )

CLar17. Nós (equipe de trabalho) não estamos autorizados a planejar nosso próprio trabalho. [R]

CLar24. Nós (equipe de trabalho) somos livres para tomar minhas próprias decisões. [R]

Estilo de equipe (10 itens: 4 excluídos e demais com cargas de 0,629 a 0,755)

CLeg4. Se nós (equipe de trabalho) solicitamos, recebemos ajuda uns dos outros.

CLeg11. Se nós (equipe de trabalho) temos uma pergunta sobre o nosso trabalho

existe alguém da nossa equipe de trabalho disponível para respondê-la.

Oportunidade de desenvolvimento (10 itens: 3 excluídos e demais com cargas de 0,635 a 0,813 )

Clod5. Existem muitas maneiras diferentes para aprender novos trabalhos na organização em que trabalhamos.

Orientações sobre como realizar o trabalho (10 itens: 4 excluídos e demais com cargas de 0,683 a 0,771 )

Clort6. Informações relevantes para o nosso trabalho são mantidas atualizadas.

Clort13. Orientações escritas sobre como realizar nosso trabalho estão disponíveis para que possamos consultar.

0,694

Satisfação com o local de trabalho (10 itens: 2 excluídos e demais com cargas de $0,613$ a 0,766$)$

Cls7. Algumas pessoas da minha equipe de trabalho não se dedicam para executar uma boa tarefa.

Cls14. Algumas pessoas da minha equipe de trabalho estão sempre reclamando sobre algo. 